\title{
The International Conference on Architectural Education in Asia (EDUARCHSIA 2017)
}

Being a knowledge-based practice, architecture strives to re-invent its relevance with its circumstances by developing a system of knowledge. For millenia, the knowledge of architecture was developed based on certainty. Vitruvian triad began with firmitas connoting the physical permanence of a building, followed by utilitas relying on the stability of function conducted in such building, while the last, its notion of venustas implies eternal beauty giving delight to its spectators.

In current situations, uncertainty is the norm, and certainty is the exception. Architecture exists in a world where so many different cultures are intermingle at the same time, each with their own value system. The knowledge of architecture faces an enormous complexity of values, interpretations, and readings.

Social un-stability, as well as massive mobility and migration prevail. Place attachment of people and their sense of identity and affiliation are constantly re-shaped in this situations. The absence of place and multiplicity of place might occur in an age-old place.

Enormous flows of information due to the advancement of digital technology alters our life fundamentally. In the meantime, information technology itself is in a perpetual transformation. From the tools being the extension of our hands to make things, it becomes the extension of our mind to vision things that we are going to produce. It opens possibilities and enables us to imagine what was previously unimaginable, realize what was unrealizable, as well as to conceive what was unconceivable. However, know, such tools became the extension or even foundation of our existence. Do we need to stay and gather on a certain place, or shall we move and wander depend on the digital existence of ourselves. Our needs, urgency and behavior are mediated, driven, or in some extents determined by this new apparatus.

Architecture in Asia with its immense diversity in complexity currently experience even more fundamental transformations in social relations, technological advancement, environmental management, and cultural appropriation. The existing configuration of knowledge is no longer adequate to comprehend and develop architecture in this region.

Former architectural inquiries on what is the shape of our buildings and how do we shape our building to suit and adapt with contemporary circumstances are still valid, but new challenges like do we still need buildings and why do we construct buildings.

This Conference calls for vision, insight, ideas and theories to re-chart the knowledge of architecture in order to inform the improvement of architectural education and practice in Asia particularly in the themes of:

\section{- Humanity and its challenges}

One of the most noble intentions of architecture is to dignify human existence on earth. Humanity, however, currently faces serious challenges due to the local and worldwide conflicts, the propagation and advancement of machine and appliances, and the proliferation of poverty. In their thinking and practice, architects should propose new ways to regain its raison d'etre to support humanity in its dignified state.

\section{- Multitude of cultural complexity}

Culture and history are not always closely related with geographical entity. Regions are fluids and overlapping. Nation states loosen their boudaries as people and capitals move from one part of the world to another massively. Vernacularism, tradition and identity as manifested themselves in architecture should be re-defined and re-invented in order to maintain their relevances with current circumstances.

\section{- Digital reality and digital technology}

More than a tool to extend our hands, information and communication technology has been developed into extend our ways of conceiving the world and our ways of changing the world. Digital technology becomes digital reality as virtuality is transformed into augmented reality. Form and function-being the fundamentals of architecture are no longer conceived as in the previous architectural thinking. Fluidity, transience and instability of forms and functions become the norm to cope with uncertainty of the world.

\section{- Environmentally appropriate technology}

Environment is in the state of peril due to the massive industrialization and urbanization. This situation causes in increasing consumption of resources and proliferating negative impacts to the environment. Architecture should propose a breakthrough in the formulaition of its knowledge in order to lessen the depletion of nature.

- Uncertainty of urbanism and territoriality

Since 2007 more people live in urban are than in other places. The urban areas where most of us live are not the same kind of place as before. Urbanism and architecture previously relies on the stability of space and other geographic entities. Centers virtually disappear and distributed network is more prevalent than centralized hierarchy. 
Boundaries are dissolved and spatial entities are alwys in the state of change. Architecture needs a new way of thinking to understand of the ever-changing space and its challenges, problems and opprtunities in order to be able propose improvements. 